\title{
Unconventional Surface Plasmon Excitations in $\mathrm{Bi}_{2} \mathrm{Se}_{3}$
}

\author{
Cigdem Ozsoy-Keskinbora ${ }^{1}$, Nahid Talebi ${ }^{1}$, Hadj M. Benia ${ }^{2}$, Christoph T. Koch ${ }^{3}$, Peter A. van Aken ${ }^{1}$
}

1 Stuttgart Center for Electron Microscopy (StEM), MPI for Intelligent Systems, Stuttgart, Germany.

2 Nanoscale Science, MPI for Solid States Research, Stuttgart, Germany.

3 Institute for Experimental Physics, Ulm University, Ulm, Germany.

The investigation of surface plasmon excitations has recently become a field of research due to their high potential to be applicable in sensors ${ }^{1}$, information technologies ${ }^{2}$, cancer research ${ }^{3}$ etc. These coherent delocalized electron oscillations are common at metal-dielectric interfaces. However, they also exist in highly doped semiconductors, conducting oxide system or graphene, i.e. in many other systems with high carrier mobility. This poses the question whether such resonances can also be observed at the insulator interfaces.

Bismuth Selenide is an insulator material which has been investigated due to its thermoelectric behavior for a long time. In 2009, it became popular after being identified as a topological insulator, which means that it behaves as an insulator in the bulk and metallic at the surface. This also makes $\mathrm{Bi}_{2} \mathrm{Se}_{3}$ a potential candidate for surface plasmons resonances. Dirac plasmons in $\mathrm{Bi}_{2} \mathrm{Se}_{3}$ were first observed in 2013 with energy in the range of $0.5-1 \mathrm{eV}^{4}$.

The Dirac state is not the only reason for the existence of a plasmon resonance in $\mathrm{Bi}_{2} \mathrm{Se}_{3}$. The highly anisotropic tetradymites crystal structure has also highly anisotropic dielectric properties ${ }^{5}$ allowing plasmon excitation. In this study we would like to show energy filtered transmission electron microscopy (EFTEM) and a finite-difference frequency-domain (FDTD) study for investigating $\mathrm{Bi}_{2} \mathrm{Se}_{3}$ nanoplates and try to find an explanation for plasmon excitations.

The EFTEM study was carried out using the 200 kV FEG-TEM Sub-Electron-Volt-Sub-ÅngstromMicroscope (Zeiss SESAM) equipped with an electrostatic monochromator and the in-column MANDOLINE filter with a $0.2 \mathrm{eV}$ slit width. As shown in Fig. 1 localized excitations exist at the surface of the $\mathrm{Bi}_{2} \mathrm{Se}_{3}$ crystal and featuring collective modes at different energies. These results supported by FDTD simulations to be able to explain the interplay between edge plasmons and surface plasmons which together make up the total electron energy loss signal shown in Fig.2.

\section{References:}

1. Wang, Z et al, Analytical Chemistry, 86 (2013), 1430-1436.

2. Kosmeier, S. et al, Scientific Reports. 3 (2013), 1808.

3. Cai, W.; Gao, T.; Hong, H.; Sun, J., Science and Applications 2008, 17-32.

4. Di Pietro et al, Nat Nano 8 (2013),556-560.

5. Esslinger, M. et al, ACS Photonics 1 (2014), 1285-1289. 

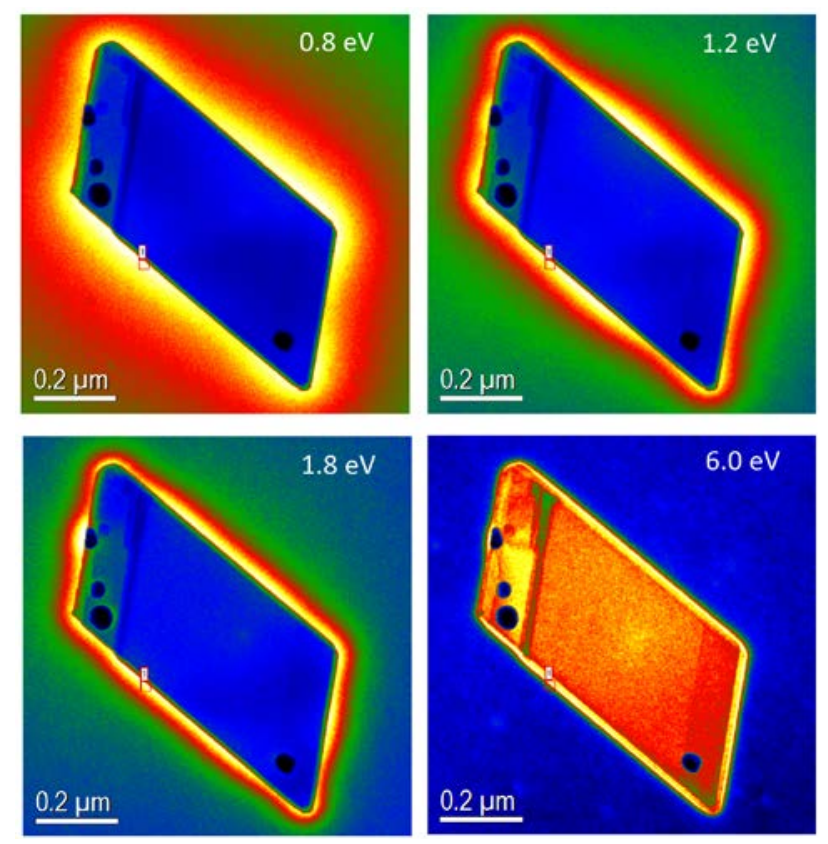

Figure 1. EFTEM images which shows of localized plasmons excitation in a $\mathrm{Bi}_{2} \mathrm{Se}_{3}$ nanoplates.

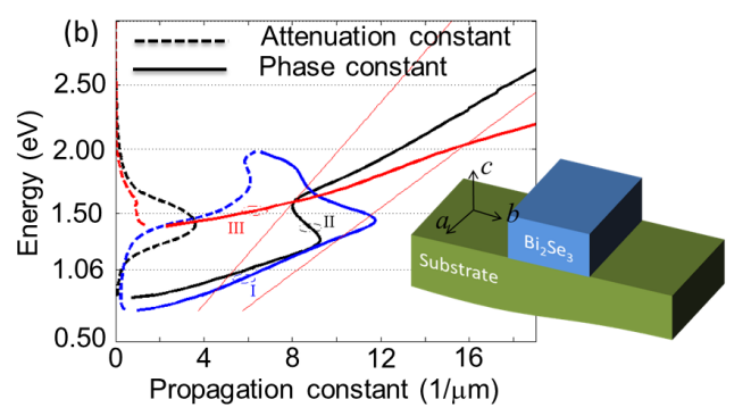

Figure 2. Propagation constant of plasmon polaritons propagating in a channel waveguide made of Bi2Se3. 